\title{
Testing Patience on Patients
}

Testing patience on patients has lost its edge no longer do they feel the pain of it all the elderly and the ill just linger in wait for the impending hands of death.

The off-white walls reflect the off faces hoping for a cure the chairs can no longer support those with sullen faces.

Hygiene and personal care faded away matted hair and five day worn underwear, sweat patches become the norm dirt hides in wrinkles and spots.

Feeling long gone for life illness sapped bodies sit in wait for an extension to tolerate another day. 